\title{
High accuracy determination of the shape of the efficiency curve of the HPGe detector in the energy range 900 to $1300 \mathrm{keV}$
}

\author{
Ayman Ibrahim Hawari *, Ronald F. Fleming \\ The Untversity of Michlgan, Department of Nuclear Engineering, Ann Arbor, MI 48109, USA
}

\begin{abstract}
A method for high accuracy characterization of the shape of a High Purity Germanium (HPGe) detector efficiency curve has been developed. Radionuclides with decay schemes having at least two $\gamma$ lines, for which the yield ratio can be calculated accurately, are used to establish the shape of the detector efficiency curve. Measurements using ${ }^{60} \mathrm{Co}$ and ${ }^{46} \mathrm{Sc}$ sources show that the behavior of the efficiency curve of the detector on a log-log scale is indistinguishable from that of a straight line with a slope of $-0.828 \pm 0.004$. Measurements using a ${ }^{24} \mathrm{Na}$ source show that above $1368 \mathrm{keV}$ this behavior no longer holds due to pair production and escape effects. The measured value of the slope in that case is $-0.951 \pm 0.002$. The proposed method is to be used in measurements requiring the minimum calibration bias in the determination of the reaction rate ratios, such as reactor dosimetry applications, integral cross section measurements and $k_{0}$ determination for neutron activation analysis.
\end{abstract}

\section{Introduction}

Many nuclear measurements require accurate knowledge of the shape of the efficiency curve of a High Purity Germanium Detector (HPGe) in a particular energy range, but do not require that the magnitude of the efficiency be known. Examples are measurements which require the determination of reaction rate ratios such as reactor dosimetry applications, integral cross section measurements and $k_{0}$ determination for neutron activation analysis. The method proposed in this paper is based upon the fact that for certain nuclides which decay by emission of at least two gammas, the ratio of the gamma yields can be calculated accurately from the decay schemes.

\section{Shape of the detector efficiency curve}

The purpose of this work is to establish a method that would produce accurate efficiency ratios for an HPGe in a limited energy range. The net photopeak counts for a particular gamma line is

$C_{\imath}=\epsilon_{\imath} \Gamma_{\imath} \frac{A_{0}}{\lambda}\left(1-\mathrm{e}^{-\lambda \tau}\right)$,

\footnotetext{
* Corresponding author.
}

where

$C_{1}=$ net counts from the $l$ th $\gamma$ line,

$\epsilon_{l}=$ photopeak efficiency for the $i$ th $\gamma$ line,

$\Gamma_{\imath}=$ the decay probability per decay of the parent for the $i$ th line,

$\mathrm{A}_{0}=$ activity of the source at the start of a count,

$\lambda=$ decay constant of the radionuclide,

$\tau=$ counting time.

Using Eq. (1) the ratio of the counts for any two $\gamma$ lines from the same source reduces to

$$
\frac{C_{1}}{C_{2}}=\frac{\epsilon_{1} \Gamma_{1}}{\epsilon_{2} \Gamma_{2}}
$$

so that the relative efficiency is

$$
\frac{\epsilon_{1}}{\epsilon_{2}}=\frac{C_{1} \Gamma_{2}}{C_{2} \Gamma_{1}} .
$$

The uncertainty in a measured efficiency ratio is due to the uncertainty in the ratio of the net counts and the uncertainty in the $\gamma$ yield ratio. If a radionuclide has a sufficiently well known decay scheme that the yield ratio can be determined accurately, the efficiency ratio can be determined to the precision of the count ratio.

Decay schemes were evaluated to identify nuclides for which the $\gamma$ yield ratio could be calculated from basic principles with sufficient accuracy. Five nuclides appear to satisfy these requirements. ${ }^{24} \mathrm{Na},{ }^{46} \mathrm{Sc},{ }^{48} \mathrm{Sc},{ }^{60} \mathrm{Co}$ and ${ }^{94} \mathrm{Nb}$. Results are presented here for ${ }^{60} \mathrm{Co},{ }^{46} \mathrm{Sc}$ and ${ }^{24} \mathrm{Na}$. 


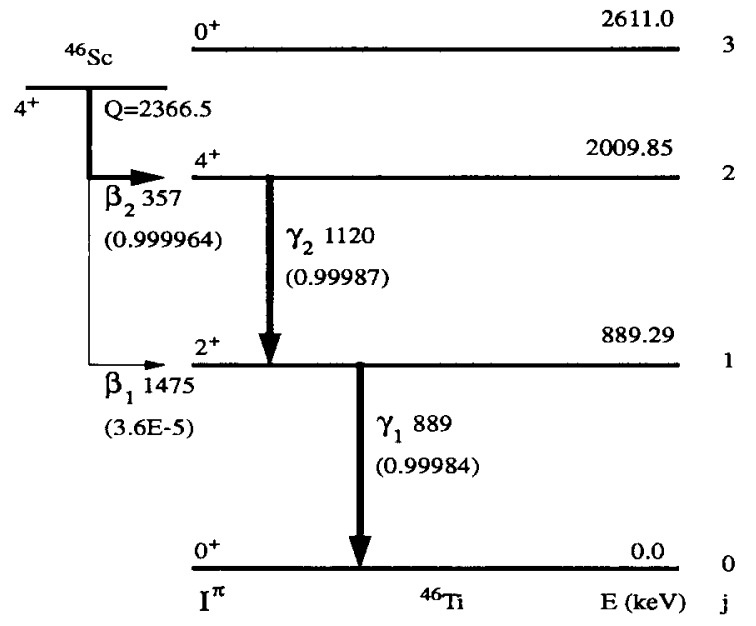

Fig. 1. The ${ }^{46} \mathrm{Sc}$ decay to levels of ${ }^{46} \mathrm{Ti}$.

For ${ }^{46} \mathrm{Sc}$, following Fig. 1, the $\gamma$ yield ratio is given by

$\frac{\Gamma_{1}}{\Gamma_{2}}=\left(\frac{1+\alpha_{2}}{1+\alpha_{1}}\right)\left(1+\frac{P\left(\beta_{1}\right)}{P_{12}}\right)$,

where

$\alpha_{i} \quad=$ internal conversion coefficient for the $i$ th line,

$P(\beta)=$ the $\beta$ decay probability to state $j$ per decay of the parent,

$P_{t} \quad=$ the $i$ th transition probability, where $\Gamma_{t}=P_{t} /(1$ $\left.+\alpha_{1}\right)$.

$P_{J J^{\prime}} \quad=$ the tansition probability from state $j$ to state $j^{\prime}$ per decay of the parent, where $\Gamma=P /(1+\alpha)$.

For ${ }^{60} \mathrm{Co}$, following Fig. 2 , the $\gamma$ yield ratio is given by

$\frac{\Gamma_{1}}{\Gamma_{2}}=\left(\frac{1+\alpha_{2}}{1+\alpha_{1}}\right)\left(1+\frac{P\left(\beta_{1}\right)+P_{21}+P_{31}+P_{51}}{P_{41}}\right)$.

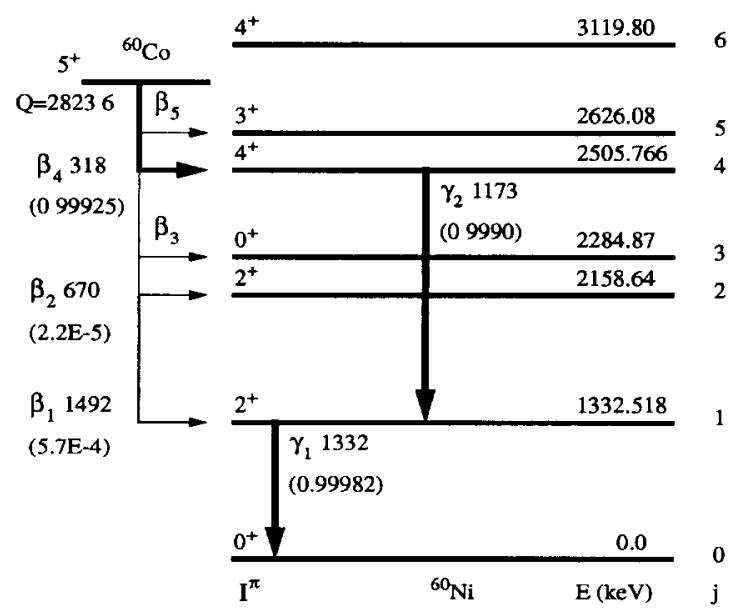

Fig. 2. The ${ }^{60}$ Co decay to levels of ${ }^{60} \mathrm{Ni}$.
Table 1

Literature and evaluated quantities

\begin{tabular}{|c|c|c|c|c|}
\hline Source & $E_{1}(\mathrm{keV})$ & $E_{2}(\mathrm{keV})$ & $\Gamma_{1} / \Gamma_{2}$ & $\mathrm{n}_{0}$ \\
\hline${ }^{60} \mathrm{Co}$ & 1332.501 & 1173.237 & $\begin{array}{l}1.00069 \\
\pm 0.00022\end{array}$ & $\begin{array}{l}-0.005 \\
\quad \pm 0.002\end{array}$ \\
\hline${ }^{46} \mathrm{Sc}$ & 889.277 & 1120.545 & $\begin{array}{l}0.999971 \\
\pm 0.000018\end{array}$ & $\begin{array}{c}-0.00013 \\
\pm 0.00008\end{array}$ \\
\hline
\end{tabular}

The same approach was used to evaluate the ratio for ${ }^{24} \mathrm{Na}$. The results are presented in Table 1 along with their uncertainties and related data obtained from the literature [1-5].

To test the ideas presented above, a power law model for the efficiency curve of an HPGe detector was chosen, $\epsilon \propto E^{-m}$,

where $\mathrm{E}$ is the $\gamma$-ray energy and $m$ is an empirical constant representing the slope of the efficiency vs. energy curve on a $\log -\log$ scale [6]. Hence, for two distinct $\gamma$ lines the ratio of the efficiencies can be written as

$\frac{\epsilon_{1}}{\epsilon_{2}}=\left(\frac{E_{2}}{E_{1}}\right)^{m}$.

Combining Eqs. (3) and (7) yields

$\left(\frac{E_{2}}{E_{1}}\right)^{m}=\frac{C_{1}}{C_{2}} \frac{\Gamma_{2}}{\Gamma_{1}}$.

Solving this for $m$ yields

$m=\frac{\ln \left(C_{1} / C_{2}\right)-\ln \left(\Gamma_{1} / \Gamma_{2}\right)}{\ln \left(E_{2} / E_{1}\right)}$.

Eq. (9) can also be written as

$m=n-n_{0}$

$n=\ln \left(C_{1} / C_{2}\right) / \ln \left(E_{2} / E_{1}\right)$,

$n_{0}=\ln \left(\Gamma_{1} / \Gamma_{2}\right) / \ln \left(E_{2} / E_{1}\right)$.

Using Eq. (10) the power $m$ can be obtained by measuring $n$ and evaluating $n_{0}$ from the literature, with Eqs. (4) and (5). The uncertainty in $m$ is due to the uncertainties in $n$ and $n_{0}$. The uncertainty in $E_{2} / E_{1}$ is less than 1 part in $10^{6}$ and can be neglected. The uncertainty in $n$ due to counting statistics is given by

$\sigma_{n}=\left(\frac{\sigma_{C_{1} / C_{2}}}{C_{1} / C_{2}}\right) / \ln \left(E_{2} / E_{1}\right)$.

The uncertainty in $n_{0}$ is due to the uncertainty in the $\Gamma_{1} / \Gamma_{2}$ ratio. The uncertainty in $m$ reported here is that due to counting statistics only, that is $\sigma_{m}=\sigma_{n}$.

The efficiency ratio $\epsilon_{l} / \epsilon_{j}$ for any two energies $E_{l}$ and $E_{J}$ is then determined by measuring $m$ and applying Eq. 
(7). A bound on the uncertainty in the efficiency ratio is given

$B=\left|\frac{\left(E_{\jmath} / E_{\imath}\right)^{m+b \sigma_{m}}}{\left(E_{j} / E_{\imath}\right)^{m}}-1\right|=\left|\left(\frac{E_{\jmath}}{E_{\imath}}\right)^{b \sigma_{m}}-1\right|$,

where $\sigma_{m}$ is the uncertainty in $m$ and $b$ is a numerical multiplier.

The suggested method can also be used to evaluate the gamma yield ratio for a nuclide emitting two gammas in the energy range where the efficiency ratio is known. This ratio is given by

$$
\frac{\Gamma_{1}}{\Gamma_{2}}=\frac{C_{1} / C_{2}}{\left(E_{2} / E_{1}\right)^{m}}
$$

and its uncertainty is given by the random uncertainty in the count ratio and the systematic bias due to the uncertainty in $m$. This bias is the same $B$ given in Eq. (12).

\section{Experimental determination of $m$}

Sources of approximately $1 \mu \mathrm{Ci}$ activity were prepared by irradiation in the Ford Nuclear Reactor at the University of Michigan. Disks, $1 / 16$ in. in diameter and 1-5 mils in thickness, were prepared for each element. The sources were counted using an HPGe and at a distance of $10 \mathrm{in}$. from the detector surface. The HPGe was a closed end coaxial detector with a diameter of $52.5 \mathrm{~mm}$, a length of $46 \mathrm{~mm}$ and an active volume of $93.6 \mathrm{~cm}^{3}$. Several spectra were collected from each source. The full energy peaks were integrated and background corrections applied to give the net counts for each peak [7]. Applying Eqs. (10) and (11) provided a value of $n$ and of $\sigma_{n}$ for each individual spectrum. The weighted mean of the values of $n$ for all the spectra was taken to represent the value of $n$ over the energy range covered by the $\gamma$ lines of the radionuclide. As can be seen from Table 2 the values of the power $m$ obtained for the ${ }^{46} \mathrm{Sc}$ and ${ }^{60} \mathrm{Co}$ sources are statistically indistinguishable, implying that the slope of the efficiency curve in this range can be taken to be the weighted mean of these values and is $-0.828 \pm 0.004$. On the other hand, the value of the slope for the ${ }^{24} \mathrm{Na}$ source is, $-0.951 \pm$ 0.002 which is clearly different indicating a different shape of the efficiency curve above $1368 \mathrm{keV}$. This behavior is attributed to the pair production and escape effects of the $2754 \mathrm{keV}$ line of ${ }^{24} \mathrm{Na}$.

Table 2

Measured values of $m$ using the power law model

\begin{tabular}{llll}
\hline Source & $C_{1} / C_{2}$ & $n$ & $m$ \\
\hline${ }^{60} \mathrm{Co}$ & $0.900 \pm 0.001$ & $0.827 \pm 0.008$ & $0.832 \pm 0.008$ \\
${ }^{46} \mathrm{Sc}$ & $1.211 \pm 0.001$ & $0.827 \pm 0.004$ & $0.827 \pm 0.004$ \\
Mean $m$ & - & - & $0.828 \pm 0.004$ \\
\hline
\end{tabular}

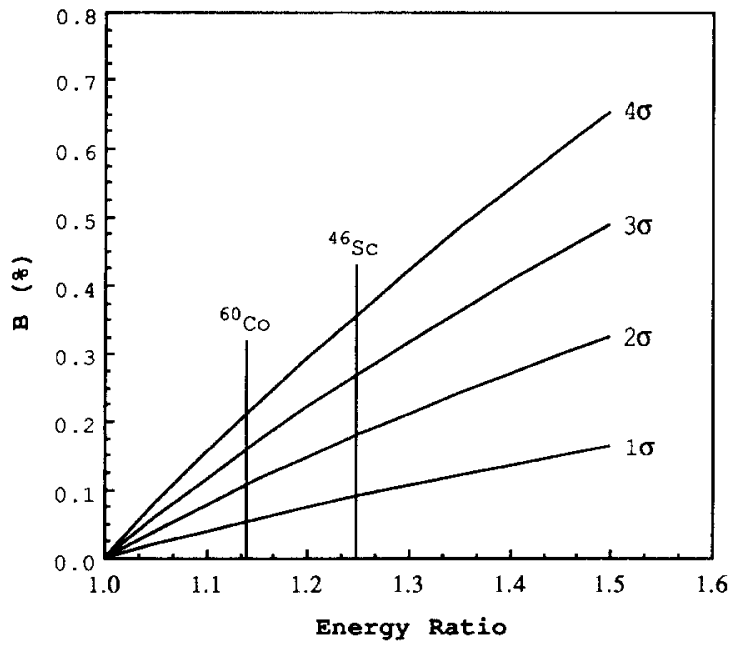

Fig. 3. The uncertainty in the efficiency ratio as a function of the energy ratio. The energy range extends from 889 to $1332 \mathrm{keV}$.

The uncertainty in the efficiency ratio for any two $\gamma$ energies in the energy range was calculated using Eq. (12). As shown in Fig. 3 the uncertainty in the efficiency ratio is less than 0.005 for a $3 \sigma$ confidence limit. For a $1 \sigma$ confidence limit the uncertainty in the efficiency ratio is less than 0.001 over most of the energy range.

\section{Conclusions}

A method for characterizing the shape of the efficiency curve of High Purity Germanium (HPGe) detectors is established. The results indicate that for a given HPGe detector the shape of the efficiency curve is indistinguishable from a straight line in the energy range extending from the energy of the lowest ${ }^{46} \mathrm{Sc}$ line at $889 \mathrm{keV}$ to the highest ${ }^{60} \mathrm{Co}$ line at $1332 \mathrm{keV}$. Above $1332 \mathrm{keV}$ results show a deviation from this behavior as demonstrated by the ${ }^{24} \mathrm{Na}$ measurements. Using this method of calibration, the efficiency ratio for any two $\gamma$ lines in this energy range can be found to within $0.1 \%$.

\section{References}

[1] P.M. Endt, Nucl. Phys. A 521 (1990) 1.

[2] Nucl. Data Sheets 49 (1986) 289.

[3] Nucl. Data Sheets 48 (1986) 268.

[4] Nucl. Data Sheets 68 (1993) 288

[5] Nucl. Data Sheets 69 (1993) 25.

[6] K Debertin and R.G. Helmer, Gamma- and X-Ray Spectrometry with Semiconductor Detectors (North-Holland-Elsevier, New York, 1988).

[7] R.M. Lindstrom, Proc Int Conf. on Nuclear Analytical Methods in the Life Sciences, Prague, September 1993. 